Discrete Comput Geom 28:223-229 (2002)

DOI: $10.1007 / \mathrm{s} 00454-002-2792-6$

\title{
Triangulated Spheres and Colored Cliques
}

\author{
Ron Aharoni, Maria Chudnovsky, and Andrě̆ Kotlov \\ Department of Mathematics, Technion, \\ Haifa 32000, Israel
}

\begin{abstract}
In [1] a generalization of Hall's theorem was proved for families of hypergraphs. The proof used Sperner's lemma. In [5] Meshulam proved an extension of this result, using homology and the nerve theorem. In this paper we show how the triangulations method can be used to derive Meshulam's results. As in [1], the proof is based on results on extensions of triangulations from the sphere to the full ball. A typical result of this type is that any triangulation of the $(d-1)$-dimensional sphere $S^{d-1}$ can be extended to a triangulation of the ball $B^{d}$, by adding one point at a time, having degree at most $2 d$ to its predecessors.
\end{abstract}

\section{Introduction}

Recently Haxell and the first author of this paper [1] generalized Hall's theorem, which concerns families of sets, to families of hypergraphs. The generalization provides a sufficient condition for the existence of systems of disjoint representatives (SDRs) in families of hypergraphs. The proof used Sperner's lemma and the existence of particular triangulations of the $n$-dimensional simplex, satisfying certain special conditions.

Later, Meshulam [5] proved an extension of this result, an interpolation between it and another known sufficient condition for the existence of SDRs (implicitly proved in [4]). His proof used homology and the nerve theorem.

The present paper re-proves Meshulam's results, using again the more elementary approach of triangulations.

The lemma used in [1] for the inductive construction of the special triagulations was:

Lemma 1.1. Any triangulation $T$ of the sphere $S^{d-1}$ can be extended to a triangulation $T^{\prime}$ of the ball $B^{d}$, such that the points of $T^{\prime} \backslash T$ all lie in the interior of the ball, and each of them is connected to at most $d$ points of $T$. 
The main tool used in the present paper is a family of extensions of this lemma. These are results concerning the extendability of triangulations from the sphere to the ball, in a way which maintains certain degree conditions on the points added. Lemma 1.1 is at one end of the spectrum of these results. At the other end of the spectrum is:

Lemma 1.2. Any triangulation $T$ of the sphere $S^{d-1}$ has an extension to a triangulation $T^{\prime}$ of the ball $B^{d}$, obtained by adding one point in the interior of the ball at a time, having degree at most $2 d$ to its predecessors.

We start with a few notions from the theory of simplicial complexes. We often use complexes and graphs in the same context, and, hence, to avoid confusion, we call the vertices of complexes "points" (so that the word "vertex" is reserved for graphs). In particular, the vertices of a simplex are called its extreme points.

For a simplicial complex $C$ we denote by $\|C\|$ its underlying space. For a complex $C$ and a point $x$ we write $C * x$ for the join of $C$ and $x$, namely, the complex $C \cup\{\sigma \cup\{x\}: \sigma \in$ $C$ \}. Given a complex $C$ and a point $v$ in it, the link of $v$ in $C$, denoted by $l k_{C}(v)$, is the set of simplices in $C$ not containing $v$, whose union with $v$ belongs to $C$.

As is well known (see, e.g., [2]), if $C$ is a triangulation of a sphere $S^{d}$, then $l k_{C}(v)$ is a triangulation of $S^{d-1}$ for any vertex $v$ of $C$.

A coloring of points of a subdivision $T$ of the $m$-dimensional simplex $\Sigma^{m}$ is called a Sperner coloring of $T$ if the extreme points of $\Sigma^{m}$ are assigned different colors, and for every $\tau \in \Sigma^{m}$ the points in $T \cap\|\tau\|$ are assigned colors from among those given to the extreme points of $\tau$. Sperner's lemma [3] asserts that in such a coloring there always exists a simplex belonging to $T$ whose extreme points are assigned different colors.

\section{Extending Triangulations of the Sphere}

In this section we prove a family of common generalizations of Lemmas 1.1 and 1.2. These generalizations interpolate between the two lemmas, in a way analogous to the interpolation of [5] between two conditions for connectivity of the clique complex of a graph.

For any $t$ we denote by $\Omega^{t}$ the graph on $2(t+1)$ vertices, which is the complement of a perfect matching. We also refer to $\Omega^{-1}$-it is the empty graph, namely, having no vertices.

Let $T$ be a triangulation of $S^{d-1}$. Consider a sequence of complexes $T=T_{0} \subset T_{1} \subset$ $\cdots \subset T_{m}=T^{\prime}$, such that $T^{\prime}$ is a triangulation of the ball $B^{d}$, and $T_{i}$ is obtained from $T_{i-1}$ by the addition of one point $v_{i}$ in the interior of the ball. For each $0<i \leq m$ we say that an edge $\left(v_{i}, u\right)$ is $t$-permissible if $u \notin T$ and the link of $\left(v_{i}, u\right)$ in $T_{i}$ contains a copy of $\Omega^{t-1}$. (Thus, for example, every edge disjoint from $T$ is 0 -permissible.)

We say that the sequence $T=T_{0} \subset T_{1} \subset \cdots \subset T_{m}$ is $(k, t)$-filling if each $v_{i}$ is connected by at most $k$ non-t-permissible edges to points of $T_{i-1}$. If there exists a $(k, t)$-filling sequence starting with $T$, we say that $T$ is $(k, t)$-fillable.

Since every interior edge is 0 -permissible, being $(k, 0)$-fillable means being extendable to a triangulation of $B^{d}$ by adding points in the interior, each connected to at most $k$ points of $T$. Since no edge is $d$-permissible in a sequence of complexes as above, $T$ 
being $(k, d)$-fillable means that it can be extended to a triangulation of $B^{d}$ by adding one point at a time, having degree at most $k$ to its predecessors.

Thus Lemmas 1.1 and 1.2 are both special cases of the following:

Lemma 2.1. For any $t \leq d$, every triangulation of $S^{d-1}$-sphere is $(d+t, t)$-fillable.

Proof. The proof is by induction on $d+t$. The basis of induction, the case $d+t=1$, is trivial. For the step of induction, fix $d+t \geq 2$ and a triangulation $T$ of $S^{d-1}$. We extend $T$ to a triangulation of $B^{d}$ in two stages. In the first we triangulate a shell of the ball, having $T$ and (say) $R$ as triangulations of its two boundaries. What is then left for the second stage is to extend $R$ to a triangulation of the "inner" ball. The advantage is then that all points of $R$ are interior points of the original ball, making easier the application of the definition of $t$-permissibility.

Stage I: Triangulating a shell. Let $v$ be a point of $T$. Its link $L$ in $T$ is a triangulation of $S^{d-2}$. Thus we can apply to it the induction hypothesis with parameters $d-1$ and $t$, which yields the existence of a sequence $L_{j}(0 \leq j \leq p)$ of complexes, each obtained from its predecessor by the addition of a point $u_{j}$, and such that (1) $L_{0}=L, L_{p}$ is a triangulation of $B^{d-1}$, and (2) $u_{j}$ is joined by at most $d-1+t$ non-t-permissible edges to points in $L_{j-1}$. Let $L_{j}^{\prime}=L_{j} * v \cup T$.

The addition of the edge $\left(u_{j}, v\right)$ adds 1 to the degree of $u_{j}$ in $L_{j}^{\prime}$ relatively to its degree in $L_{j}$. Note that any $t$-permissible edge in the sequence

$$
L_{0} \subset L_{1} \subset \cdots \subset L_{p}
$$

remains $t$-permissible for the sequence

$$
L_{0}^{\prime} \subset L_{1}^{\prime} \subset \cdots \subset L_{p}^{\prime} .
$$

Hence each $L_{j}^{\prime}$ is obtained from its predecessor by adding a point connected to at most $d+t$ non- $t$-permissible edges to points in $L_{j-1}^{\prime}$.

The complex $L_{p}^{\prime}$ consists of $T$ together with a "cap" having $v$ as its tip, triangulated by $L_{p} * v$. The complex $L_{p}^{\prime}-v$ obtained by "excising" this cap is a triangulation of $S^{d-1}$. Its points are those of $T$, with $v$ deleted and with some new points added in the interior of the sphere triangulated by $L$ (see Fig. 1).

We now choose a point of $L_{p}^{\prime}-v$ belonging to $T$, and in a similar manner excise a triangulated cap having it as a tip. In finitely many steps of this type we eliminate all
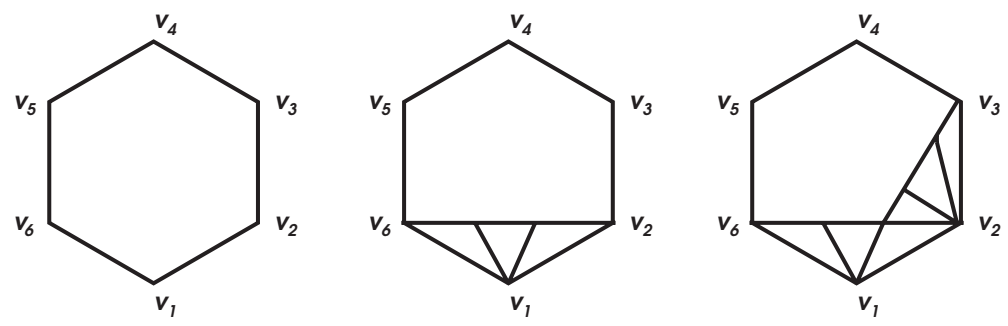

Fig. 1. Excision of caps with tips $v_{1}$ and $v_{2}$. 
points of $T$. The union of all the excised caps is then a triangulation of a shell, having $T$ as one boundary and a triangulation $R$ disjoint from $T$ as the other boundary.

What remains now is to triangulate the ball inside $R$.

Remark. In the case of $t=0$, namely, in that of Lemma 1.1, the remaining task is easy. Any triangulation of the ball will do, since the only conditions are on the degree of interior points to the points of $T$.

Stage II: Triangulating the inner ball. Pick a point $z$ in the interior of the ball bounded by $R$ (namely, in the bounded component of $\mathbb{R}^{d} \backslash\|R\|$ ). Let $v$ be a point of $R$. The link $M$ of $v$ in $R$ is a triangulation of $S^{d-2}$. By the inductive hypothesis, with parameters $d-1$ and $t-1$, there exists a sequence $M_{j}(0 \leq j \leq q)$ of complexes, each obtained from its predecessor by the addition of a point $u_{j}$, and such that (1) $M_{0}=M, M_{q}$ is a triangulation of $B^{d-1}$, and (2) $u_{j}$ is joined by at most $d-1+t-1=d+t-2$ non- $(t-1)$-permissible (for the sequence $M_{k}$ ) edges to points in $M_{j-1}$. Let $M_{j}^{\prime}=M_{j} * v \cup\left(M_{j}-M\right) * z \cup T$.

Let $\left(u_{j}, x\right)$ be a $(t-1)$-permissible edge for the sequence $M_{k}(0 \leq k \leq q)$. By the definition of permissibility, this means that its link in $M_{j-1}$ contains a copy $X$ of $\Omega^{t-2}$. Since all points of $X$ are connected in $M_{j}^{\prime}$ to $v$ and to $z$, the complex $X * v \cup X * z$ is a copy of $\Omega^{t-1}$, contained in the link of $\left(u_{j}, x\right)$ in $M_{j-1}^{\prime}$. This means that $\left(u_{j}, x\right)$ is $t$-permissible for the sequence $M_{k}^{\prime}(0 \leq k \leq q)$.

Thus all non- $t$-permissible edges connecting $u_{j}$ to points in $M_{j-1}$ for the sequence $M_{k}^{\prime}$ are also non- $(t-1)$-permissible for the sequence $M_{k}$, and hence there are at most $d+t-2$ of them. Together with the two edges joining $u_{j}$ to $v$ and to $z$, this gives at most $d+t$ non- $t$-permissible edges connecting $u_{j}$ to points in $M_{j-1}^{\prime}$.

The complex $M_{q}^{\prime}$ is similar to the complex named $L_{p}^{\prime}$ in the first stage: it consists of $T$ together with a cap having $v$ as its tip, triangulated by $M_{q} * v$. The difference is that there is added to it the cone $\left(M_{q}^{\prime}-M\right) * z$.

We now pick a point in $M_{q}^{\prime} \cap T$, and repeat the procedure to "excise" a cap having it as a tip. This is done just as in stage I, the difference being, again, that the points added are connected also to $z$.

As in stage I, once all points of $T$ are eliminated, a shell has been triangulated, one of its boundaries being $R$. Let $W$ be its other (inner) boundary. Then in the complex obtained, $z$ is connected to all points of $W$. This means that the complex obtained is a triangulation of the ball $B^{d}$.

\section{Meshulam's Theorem}

In this section we show how the results on extendability of triangulations imply a theorem of Meshulam [5]. We do not use Lemma 2.1 itself, but the steps of the proof will be the same. This part is then implemented into a proof scheme used in [1].

Throughout the rest of the paper, let $G$ be a graph on a vertex set $V$ with a loop at each vertex, so that its complement, $H$, has no loops. As usual, $G[A]$ denotes the induced subgraph of $G$ spanned by $A \subseteq V$. For a vertex $v \in V$ we denote by $\Gamma(v)$ the set of neighbors of $v$ in $G$. (Note that $v \in \Gamma(v)$ ). 
We say that $G$ is $k$-narrow with respect to a set $A$ of vertices if every $k$ vertices of $G$ has a common neighbor in $A$. If $A=V$ we say just that $G$ is $k$-narrow. (The measure of narrowness of the complement of a graph is the domination number of the graph. In [1] the domination number of the line graph of a hypergraph is called the width of the hypergraph, hence the "narrowness" terminology.)

A set $A$ of vertices is called $t$-free if $G[A]$ does not contain $\Omega^{t}$ as an induced subgraph.

A graph is called $(k, t)$-narrow if it is $k$-narrow with respect to a $t$-free set $A$. We say then that it is also $(k, t)$-narrow with respect to $A$.

The following is obvious:

\section{Observation 3.1.}

(1) If $G$ is $(k, t)$-narrow with respect to $A$, then $G[\Gamma(v)]$ is $(k-1, t)$-narrow with respect to A for any vertex $v$.

(2) If $G$ is $(k, t)$-narrow with respect to $A$, then for any pair $u, v$ of nonadjacent vertices of $A$, the graph $G[\Gamma(u) \cap \Gamma(v)]$ is $(k-2, t-1)$-narrow with respect to $A \cap \Gamma(u) \cap \Gamma(v)$.

Lemma 3.2. Let $T$ be a triangulation of $S^{d-1}$ and let $\varphi$ be a homomorphism (an edgepreserving map) from $T$ to a graph $G$. If $G$ is either $2 d$-narrow or $(d+t, t)$-narrow for some $t \leq d$, then there exist an extension $\hat{T}$ of $T$ to a triangulation of the ball $B^{d}$ and an extension $\hat{\varphi}: \hat{T} \rightarrow G$ of $\varphi$.

Proof. We prove the case that $G$ is $(d+t, t)$-narrow with respect to some set of vertices $A$. The proof of the case of $2 d$-narrowness is similar. The proof is by induction on $d+t$. We shall strengthen the inductive hypothesis, to include the condition that $\hat{\varphi}[\hat{T} \backslash T] \subseteq A$.

We construct $\hat{T}$ along the same lines as in the proof of Lemma 2.1. That is, we do it in two stages, in the first of which a shell is constructed, and in the second the ball inside the shell is filled.

Let $v$ be a point of $T$. Its link $L$ in $T$ is a triangulation of $S^{d-2}$. By Observation 3.1 $G^{\prime}=G[\Gamma(v)]$ is $(d-1+t, t)$-narrow with respect to $A \cap \Gamma(\varphi(v))$. Hence we can apply the induction hypothesis with parameters $d-1$ and $t$, to obtain a triangulation $\hat{L}$ of $B^{d-1}$ extending $L$, and a homomorphism $\theta: \hat{L} \rightarrow G^{\prime}$ extending $\varphi \mid L$. Then $\varphi \cup \theta$ is a homomorphism of $T \cup \hat{L} * v$ into $G$, where $T \cup \hat{L} * v$ is a triangulation $T^{\prime}$ of a sphere together with a cap, having $v$ as the tip. The triangulation $T^{\prime}$ may have additional points to those of $T$, but they are all inside the ball circumscribed by $T$, while it does not contain the point $v$ of $T$.

As in the proof of Lemma 2.1, we now proceed to choose a point of $T^{\prime}$ belonging to $T$, and repeat the same procedure with it replacing $v$. In finitely many steps we extend $T$ to a triangulation of a shell having $T$ as the outer boundary, and (say) $R$ as the inner boundary, and we extend $\varphi$ to the new triangulation. For convenience, we still denote the extended homomorphism by $\varphi$. Our aim is to extend $T$ to the ball circumscribed by $R$, and extend $\varphi$ to it.

To do this, we choose a point $z$ in the interior of the ball bounded by $R$, and define $\varphi(z)=a$, where $a$ is any chosen vertex in $A$. Let $Y$ be the set of points $v$ of $T$ such that $(a, \varphi(v)) \notin E(G)$, and order $Y$ as $y_{1}, y_{2}, \ldots, y_{q}$. 

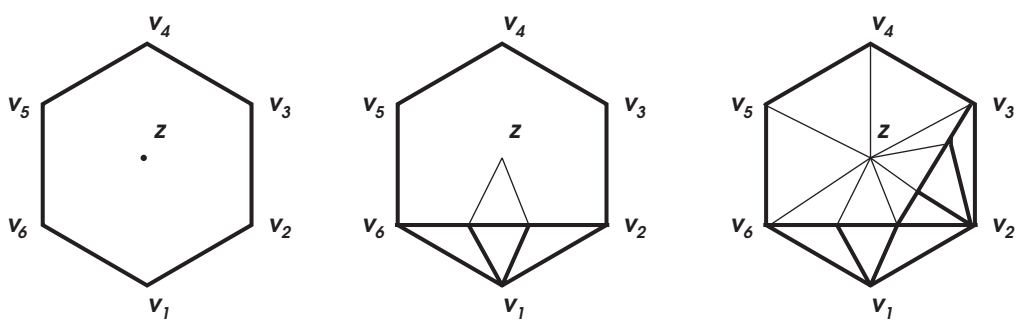

Fig. 2. Triangulation of the inner ball with $Y=\left\{v_{1}, v_{2}\right\}$.

The link $M_{1}$ of $y_{1}$ in $R$ is a triangulation of $S^{d-2}$. By Observation $3.1 G^{\prime}=$ $G\left[\Gamma\left(\varphi\left(y_{1}\right)\right) \cap \Gamma(a)\right]$ is $(d-1+t-1, t-1)$-narrow with respect to $A \cap \Gamma\left(\varphi\left(y_{1}\right)\right) \cap \Gamma(a)$. Hence we can apply the induction hypothesis with parameters $d-1$ and $t-1$, to obtain a triangulation $\hat{M}_{1}$ of $B^{d-1}$ extending $M_{1}$, and a homomorphism $\theta: \hat{M}_{1} \rightarrow G^{\prime}$ extending $\varphi \uparrow M_{1}$. Then $\varphi \cup \theta$ is a homomorphism of $T_{1}=T \cup \hat{M}_{1} * y_{1} \cup \hat{M}_{1} * z$ into $G$, where $T_{1}$ is a triangulation of a sphere together with two cones, having $y_{1}$ and $z$ as tips.

The link of $y_{2}$ in $T_{1}$ is again a $(d-2)$-sphere, and we can apply the inductive hypothesis to it as we did with $y_{1}$. In $q=|Y|$ steps we obtain a triangulation of a shell inside $R$, where the only points not connected to $z$ are those of $R-Y$. Adding all edges $(z, r), r \in R-Y$ yields a triangulation of the ball inside $R$, on which the $q$ th extension of $\varphi$ is a homomorphism into $G$, as desired (see Fig. 2).

Let $V_{1} \dot{\cup} \ldots \dot{\cup} V_{m}$ be a fixed partition of $V$ into $m$ nonempty sets. A subgraph $K$ of $G$ is colored if $K \cap V_{i} \neq \emptyset$ for all $i \in\{1, \ldots, m\}$. The following is the main result of [5].

Theorem 3.3 [5]. Suppose that for each nonempty $\sigma \subseteq\{1, \ldots, m\}$ either the graph $G_{\sigma}:=G\left[\bigcup_{i \in \sigma} V_{i}\right]$ is $2 d_{\sigma}$-narrow (where $\left.d_{\sigma}:=|\sigma|-1\right)$ or there exists $t_{\sigma}<d_{\sigma}$ such that $G_{\sigma}$ is $\left(d_{\sigma}+t_{\sigma}, t_{\sigma}\right)$-narrow. Then $G$ contains a colored clique.

Proof. The proof follows the outline of the proof in [1]. Let $\Sigma$ be the $(m-1)$ dimensional simplex, on the vertex set $X=\left\{x_{1}, \ldots, x_{m}\right\}$. Define a homomorphism $\varphi: \Sigma^{(0)} \rightarrow G$ by letting $\varphi\left(x_{i}\right)$ be any vertex in $V_{i}(1 \leq i \leq m)$.

Using Lemma 3.2 we now construct inductively triangulations $T^{k}(0 \leq k<m)$ (where $T^{0}$ is the trivial triangulation, having only the singletons $\left\{x_{i}\right\}$ as simplices) each extending its predecessor and refining $\Sigma^{(k)}$, and we extend $\varphi$ to homomorphisms (still denoted by $\varphi$ ) on $T^{k}$, such that $\varphi\left[T^{k} \cap\|\sigma\|\right] \subseteq \bigcup_{i \in \sigma} V_{i}$ for every $\sigma \subseteq\{1, \ldots, m\}$. Coloring each point $v$ of $T^{m-1}$ with color $i$ if $\varphi(v) \in V_{i}$, we get a Sperner coloring. Applying Sperner's lemma we obtain a multicolored simplex, whose image by $\varphi$ is a colored clique.

\section{Acknowledgments}

We thank Ron Adin, Tamás Fleiner, Gil Kalai, László Lovász, and Roy Meshulam for helpful discussions. 


\section{References}

1. R. Aharoni and P. Haxell: Hall's theorem for hypergraphs, J. Graph Theory 35(2) (2000), 83-88.

2. A. Björner: Topological methods, in Handbook of Combinatorics (R. Graham, M. Grötschel, and L. Lovász, editors), Elsevier and the MIT Press, Amsterdam and Cambridge, MA (1995).

3. J. Dudgundji and A. Granas: Fixed Point Theory, Polish scientific, Warsaw (1982).

4. P. E. Haxell: A condition for matchability in hypergraphs, Graphs Combin. 11(3) (1995), 245-248.

5. R. Meshulam: The clique complex and hypergraph matchings, Combinatorica 21(1) (2001), 89-94.

Received December 7, 2000, and in revised form December 25, 2001. Online publication July 24, 2002. 